\title{
Shozo Izui
}

\section{5 years of Springer Seminars in Immunopathology}

Published online: 18 October 2003

(C) Springer-Verlag is a part of Springer Sciences + Business Media 2003

This supplement has been prepared for the 25th anniversary of the founding of Springer Seminars in Immunopathology (SSI), which coincides with the 80th birthday of Peter Miescher, founding editor of our journal and still an active member of the editorial team. We thus have two excellent reasons for celebration.

During these 25 years, tremendous progress has been made in our understanding of immunopathological events. The scientific advances for the most part have been made at the basic research level, the ultimate goal being to develop new avenues of treatment for autoimmune diseases. SSI was founded with the aim of keeping clinicians up-to-date on new developments in the field of immunopathology. In fact, understanding the pathogenic mechanisms of disease is essential for setting up rational treatment regimens. The transmission to the clinician of new knowledge and progress made in this field has become increasingly difficult with the domination of molecular biology in research. In particular, clinicians sometimes find the terminology specific to molecular biology difficult to understand. For this reason, in SSI the emphasis has been on clinically oriented articles.

Twenty-five years ago, less than ten immunomodulating drugs were available to the clinician. Today, the overall therapeutic potential has dramatically increased and there are now several such drugs available that are directed towards various targets within the immune response. With this range of pharmaceutical preparations at our disposal, it has become possible to adjust treatment to the individual needs of patients.

In this supplement, Miescher, Zavota, Ossandon and Lagano present the most recent concepts of the immunological basis of these disorders. Indeed, most of the molecular events in the immune response have only been unravelled during the past 25 years. Research progresses so rapidly that most clinicians have problems keeping up with developments. Nowadays, the immune response is at the centre of clinical immunology, and understanding it is essential and indispensable for establishing rational treatment regimens. Treatment of autoimmune disorders would seem to be relatively simple since, despite being very variable in clinical expression, they are all based on an aberrant immune response. However, there are over 40 autoimmune diseases and each one is due to a different

\footnotetext{
S. Izui $(\bullet)$

Department of Pathology,University of Geneva, 1211 Geneva 4, Switzerland e-mail: Shozu.Izui@medecine.unige.ch
} 
genetically determined autoimmune process. An additional difficulty is the extreme variability in disease expression within the same disease entity, depending on genetic factors that differ from patient to patient. Furthermore, there are variable disease-triggering and disease-modifying environmental factors, which are not only relevant in the initial stages, but throughout the entire course of the disease. In this hypervariable and complex situation, which requires constant adjustment of treatment to the continuously changing needs of the patient, rigid treatment protocols can only fail. To respond to these needs, a flexible approach towards each individual patient is indispensable.

Peter Miescher has 50 years of experience in the development and application of flexible treatment protocols. Since leaving Geneva University Hospital in 1994, he has continued his clinical activities as consultant to various groups of physicians at the university clinics of Rome and Parma. I have taken the opportunity of this special supplement to ask him, in collaboration with his young colleagues and pupils, to convey his vast personal experience in the polypharmacological management of autoimmune diseases to physicians engaged in the treatment of such disorders.

We hope you will all enjoy this double anniversary edition. 